\title{
BTG1 inhibits breast cancer cell growth through induction of cell cycle arrest and apoptosis
}

\author{
RAN ZHU $^{1 *}$, SHI-TAO ZOU ${ }^{2 *}$, JIAN-MEI WAN ${ }^{3}$, WEI LI ${ }^{4}$, XIN-LI LI ${ }^{5}$ and WEI ZHU ${ }^{1}$ \\ ${ }^{1}$ School of Radiation Medicine and Protection, Medical College, Soochow University, Suzhou, Jiangsu 215123; \\ ${ }^{2}$ Oncology Institute, Wuxi Fourth People's Hospital, Wuxi, Jiangsu 214062; ${ }^{3}$ Medical College, \\ Soochow University, Suzhou, Jiangsu 215123; ${ }^{4}$ Department of General Surgery, Second Affiliated Hospital \\ of Soochow University, Suzhou, Jiangsu 215123; ${ }^{5}$ School of Public Health, Medical College, \\ Soochow University, Suzhou, Jiangsu 215123, P.R. China
}

Received June 26, 2013; Accepted July 31, 2013

DOI: $10.3892 /$ or.2013.2697

\begin{abstract}
BTG1, which belongs to the BTG/Tob family, regulates cell cycle progression in a variety of cell types and appears to play roles in inhibiting proliferation, promoting apoptosis and stimulating cellular differentiation in multiple cell types. However, it remains unclear whether BTG1 is a breast cancer suppressor gene, and the role of BTG1 in breast cancer cell growth has not yet been determined. In the present study, we observed that BTG1 was weakly expressed in human breast tumors and in breast cancer cells (MCF-7 and MDA-MB-231). In addition, we investigated the potential effects of BTG1 on breast cancer cell proliferation, cell cycle distribution and apoptosis after stable transfection with the BTG1 expression vector. We found that overexpression of BTG1 inhibited cell proliferation, induced G0/G1 cell cycle arrest and promoted apoptosis. Further investigation indicated that overexpression of BTG1 was involved in the inhibition of the expression of cell cycle-related proteins, cyclin B1 and cyclin D1, and pro-apoptotic factors, Bax and caspase-3, and was also involved in the promotion of anti-apoptotic factor Bcl-2. In vivo, animal experiments showed that tumors overexpressing BTG1 displayed a slower growth rate than the control xenografts. TUNEL end staining assay revealed that BTG1 induced tumor necrosis and apoptosis. Taken together, our data revealed that, in breast cancer cells, BTG1 inhibits cell growth through induction of cell cycle arrest and apoptosis. These results indicate that BTG1 may be used as a novel therapeutic target for human breast cancer treatment.
\end{abstract}

Correspondence to: Professor Wei Zhu, School of Radiation Medicine and Protection, Medical College, Soochow University, 199 Ren'ai Road, Suzhou Industrial Park, Suzhou, Jiangsu 215123, P.R. China

E-mail: sdzoushitao@126.com

*Contributed equally

Key words: BTG1, breast cancer, cell cycle, apoptosis, nude mice

\section{Introduction}

Breast cancer is the most common malignancy and the major cause of cancer-related mortality in women worldwide (1). Although the 5-year survival rate has greatly increased with advances in the detection and treatment, many breast cancer patients still die from tumor malignancy. Therefore, more effective methods for the prevention and treatment are greatly needed.

BTG1 was originally identified as a translocation partner of the c-MYC gene in a case of B-cell chronic lymphocytic leukemia and belongs to a family of antiproliferative genes that also includes BTG2, BTG3, TOB and TOB2 $(2,3)$. Proteins encoded by members of this gene family have been implicated in the induction of growth arrest or apoptosis in a variety of cell systems (4). Overexpression of BTG1 was found to block proliferation during normal erythroid differentiation (5) and to induce growth arrest in a B-cell lymphoma model (6). One study demonstrated that BTG1 regulates the glucocorticoid receptor (GR)-dependent transcriptional response in leukemic cells (7), which indicates that this gene is an important target for modulating therapeutic response. The BTG1 protein has no proven intrinsic enzymatic activity, but the presence of several protein interaction domains suggests that BTG1 functions either as an adaptor molecule or as a cofactor involved in transcriptional regulation. For instance, BTG1 was identified as coactivator of the homeobox transcription factor HoxB9 (8) and the transcriptional regulator CAF1 (9). Moreover, BTG1 was shown to stimulate the activity of several myogenic transcription factors as well as nuclear receptors during muscle cell differentiation (10).

BTG1 is also essentially expressed in many tumor cells and can inhibit the proliferation of a variety of cancer cells. For instance, BTG1 is expressed predominantly in quiescent cells at the G0/G1 phase transition, with levels declining as cells enter S phase (11). Exogenous expression of BTG1 was found to result in reduced proliferation with G1 arrest and/ or apoptosis in several cell types, including NIH3T3 murine fibroblasts (2,11), microglia (12) and myoblasts (13). A role for BTG1 in cellular differentiation has been proposed based on experiments showing that BTG1 expression stimulates 
myoblast differentiation (13), and that BTG1 is upregulated in leukemic cells upon treatment with chemicals that induce differentiation (14). Thus, BTG1 appears to play roles in inhibiting proliferation, promoting apoptosis and stimulating cellular differentiation in multiple cell types.

While several studies suggest that BTG1 exhibits certain characteristics of a tumor-suppressor gene, it has not been determined whether BTG1 is a breast cancer suppressor gene, and the role of BTG1 in breast cancer cell growth remains unclear. Thus, the present study was undertaken to evaluate the effect of BTG1 on breast cancer cell (MDA-MB-231 and MCF-7) proliferation, cell cycle distribution and apoptosis by creating stable transfectants and evaluating their effect on cell growth potential in vitro. We investigated changes in cell proliferation, cell cycle progression and cell apoptosis. We also evaluated changes in the expression of several proteins that are directly related to the cell cycle (cyclin B1, cyclin D1, cyclin E1 and cell division cycle gene) and cell apoptosis (Bcl-2, Bax and caspase-3). Furthermore, we examined whether these changes are also observed in vivo using nude mice. Our aim was to determine the role of the BTG1 gene in breast cancer cell growth.

\section{Materials and methods}

Cell culture and clinical specimens. Human breast cancer cell lines, MCF-7, T-47D, MDA-MB-231, MCF-10A and MDA-MB-435, were obtained from the Shanghai Cell Bank (Shanghai, China). Human normal cells EPH4, AG11132A, HBEC, HMEC were stored in our laboratory. Cells were cultured in Dulbecco's modified Eagle's medium (Gibco-BRL, Carlsbad, CA, USA) containing $10 \%$ fetal bovine serum in a humidified atmosphere with $5 \% \mathrm{CO}_{2}$ at $37^{\circ} \mathrm{C}$. Clinical specimens were obtained from the Second Affiliated Hospital of Soochow University.

Generation of stable cell lines. To generate BTG1 overexpressing stable cell lines, cDNAs for full-length human BTG1 were amplified by PCR using the following primer sequences: forward (5'-CACCATGCATCCCTTCTACACCCGG-3') and reverse (5'-TTAACCTGATACAGTCATCATATTG-3'). The full-length cDNA was cloned into XhoI and BamHI linearized plasmid vector pcDNA3 (Clontech). The control vector pcDNA3 and human BTG1 expression vector pcDNA3-BTG1 were transfected into MCF-7 and MDA-MB-231 cells using Lipofectamine 2000 (Invitrogen). Stable clones were selected in medium containing G418 $(500 \mu \mathrm{g} / \mathrm{ml})$ (Sigma). Individual clones were isolated and expanded for further characterization. The empty vector pcDNA3 was also transfected into MCF-7 and MDA-MB-231 cells and served as the control group. The expression of BTG1 was determined by RT-PCR and western blot analysis.

Cell proliferation assay. Cell proliferation was measured with MTT assay. Briefly, cells from each experimental group were plated in 96-well plates at a density of $5 \times 10^{3}$ cells, and $180 \mu \mathrm{l}$ culture medium was added to each well. The cells were incubated at $37^{\circ} \mathrm{C}$ for $1,2,3,4,5,6,7$ or 8 days at which time the cells were incubated with $100 \mu \mathrm{l}$ of MTT solution $(5 \mathrm{~g} / \mathrm{l}$; Sigma, St. Louis, MO, USA) for 2 h. The reaction was stopped by the addition of $150 \mu \mathrm{l}$ DMSO (Sigma), and the absorbance of samples at $570 \mathrm{~nm}$ was then measured. A growth curve was plotted for each sample as the log cell number vs. time, and the growth rates were derived from the slope of each growth curve. Three independent experiments were performed, and the results were used for plotting the relative growth rate with SD.

Flow cytometric analysis of cell cycle distribution and apoptosis. For cell cycle analysis, after $48 \mathrm{~h}$ of culture, cells from each experimental group were collected and digested with trypsin and fixed with $75 \%$ ice-cold ethanol at $4^{\circ} \mathrm{C}$ overnight. Cells $\left(1 \times 10^{6}\right)$ were centrifuged at $1,000 \mathrm{rpm}$ for $5 \mathrm{~min}$, and the pellets were resuspended with $100 \mu \mathrm{g} / \mathrm{ml}$ propidium iodide (Sigma) for $30 \mathrm{~min}$ in the dark before analysis. The cell cycle profiles were assayed using the FACSCan ESP flow cytometer at $488 \mathrm{~nm}$, and data were analyzed using MultiCycle software (BD Biosciences). For analysis of apoptosis, cells from each experimental group were collected and digested with trypsin and processed as described in the Annexin V-Fluorescein Isothiocynate (FITC) Apoptosis Detection Kit I manual (BD Biosciences) and analyzed by FACScan flow cytometry (BD Biosciences).

RNA isolation and reverse transcription-polymerase chain reaction $(R T-P C R)$. Total RNA from the cells of each experimental group was extracted by TRIzol (Gibco-BRL) according to the manufacturer's instructions. cDNA was generated from total RNA, using M-MLV RT (MBI; Fermentas, Waltham, MA, USA). Amplification was performed over 28 cycles consisting of $94^{\circ} \mathrm{C}$ for $30 \mathrm{sec}, 57^{\circ} \mathrm{C}$ for $30 \mathrm{sec}$ and $72^{\circ} \mathrm{C}$ for $1 \mathrm{~min}$. PCR products were separated by electrophoresis on a $1.5 \%$ agarose gel and stained with ethidium bromide to visualize the bands. To compare differences among the samples, the relative intensity of each band was normalized against the intensity of the GAPDH band amplified from the same sample. The primer sequences for the genes and expected product sizes were as follows: 5'-TGAACGGGAAGCTCACTGG-3' (sense) and 5'-TCCACCACCCTGTTGCTGTA-3' (antisense) for GAPDH (307 bp); 5'-CACCATGCATCCCTTCTACACC CGG-3' (sense) and 5'-GCTGAGCCGCCAAAAGGT-3' (antisense) for BTG1 (498 bp).

Western blot analysis. Cells from each experimental group were lysed with SDS sample buffer (80 mM Tris- $\mathrm{HCl}$, $2 \%$ SDS, $300 \mathrm{mM} \mathrm{NaCl}$ and $1.6 \mathrm{mM}$ EDTA). Cell extracts were separated using 10\% SDS-PAGE gel electrophoresis, transferred onto nitrocellulose membranes and blocked with $5 \%$ skimmed milk. Following blocking, membranes were incubated with antibodies against $\beta$-actin, BTG1, p-CDC2, cyclin D1, cyclin B1, cyclin E1, Bax, Bcl-2, caspase-3 (Santa Cruz Biotechnology) and then incubated with HRP-conjugated anti-mouse or anti-rabbit IgG antibodies (Santa Cruz Biotechnology). Protein bands were visualized with ECL solution.

In vivo studies. Four-week-old female nude mice (SPF BALB/c) were obtained from the Laboratory Animal Center of Soochow University and kept in a room at constant temperature $\left(23 \pm 2^{\circ} \mathrm{C}\right)$ and humidity (50-70\%) with a 12-h light-dark cycle. After 
A

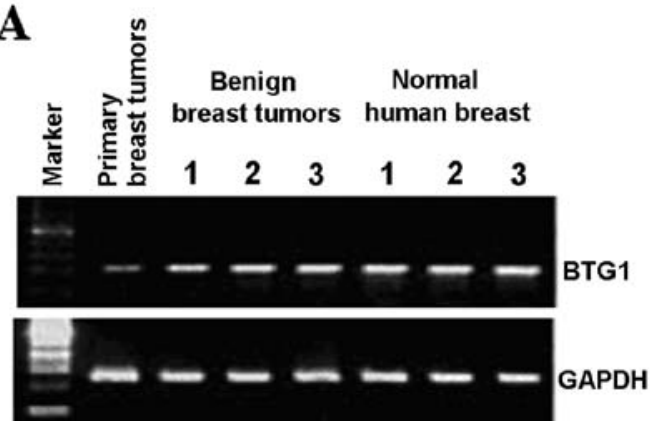

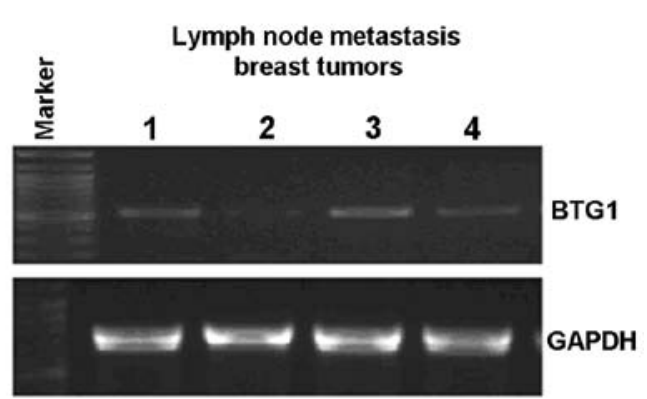

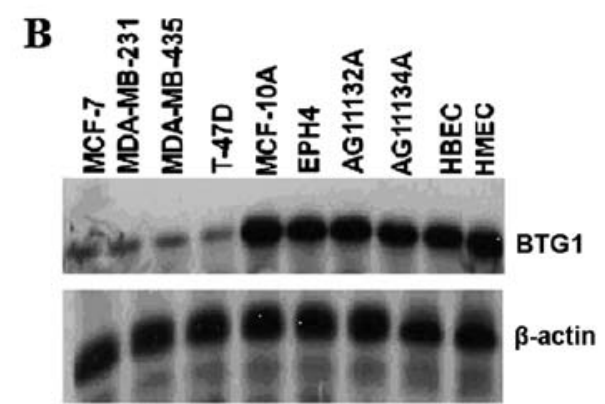

Figure 1. BTG1 expression in breast cancer cell lines and clinical specimens. (A) BTG1 mRNA was detected by RT-PCR in primary tumors, lymph node metastasis, benign breast tumors and normal human breast as indicated. GAPDH levels served as the loading control. (B) Protein expression of BTG1 was analyzed by western blot analysis in a panel of breast cancer cell lines as indicated. $\beta$-actin levels served as the loading control.

being grown to subconfluency, transfected (pcDNA3-BTG1 or negative control pcDNA3-neo) and non-treated MDA-MB-231 cells were trypsinized and harvested, washed twice with PBS and resuspended in $0.2 \mathrm{ml} \mathrm{PBS}\left(5 \times 10^{6}\right.$ cells $\left./ 0.2 \mathrm{ml}\right)$. Each group consisted of 6 nude mice. Every five days, the tumor diameter was measured, and the volume was calculated according to the formula: $\mathrm{V}=0.4 \mathrm{x}$ largest diameter $\mathrm{x}$ smallest diameter. The growth curve of each tumor was plotted, and the tumor growth ratio was calculated. Four weeks after injection of the cells, the mice were sacrificed, and the weights of the tumors were recorded and collected for histological analysis. The animal treatment protocol used in the present study was approved by the Institutional Animal Care and Use Committee.

Immunohistochemistry. The tumor xenografts were removed and fixated in $10 \%$ phosphate-buffered formaldehyde at room temperature for $48 \mathrm{~h}$, embedded in paraffin and sectioned at 4-6 $\mu \mathrm{m}$. Tumors were confirmed in H\&E-stained sections. Expression levels of molecular BTG1 and Bcl-2 were also examined using immunohistochemistry. The sections were deparaffinized, rehydrated and antigen retrieved in Tris/ EDTA buffer at $100^{\circ} \mathrm{C}$ for $15 \mathrm{~min}$ and left in the buffer for 10 min after boiling. Following a rinse in distilled water and phosphate-buffered saline (PBS), the sections were treated with $0.03 \%$ hydrogen peroxide for $5 \mathrm{~min}$ to block endogenous peroxidase activity, before incubation with mouse anti-human BTG1 and Bcl-2 antibody (Santa Cruz Biotechnology), diluted 1:100 for $30 \mathrm{~min}$ at room temperature. Negative controls were set by replacement of the primary antibody with normal polyclonal mouse $\operatorname{IgG}$ of the same subclass and concentration. Following a rinse in PBS, the sections were incubated with labeled HRP-conjugated anti-mouse antibody for $30 \mathrm{~min}$ at room temperature, and then rinsed two times in PBS before a 10-min incubation with diaminobenzene. After rinsing they were counterstained with hematoxylin, rinsed, dipped briefly in a water bath containing several drops of ammonia, before dehydration and mounting in Diatex. The stained sections were reviewed and scored using a Nikon microscope to visualize cytoplasmic and nuclear staining of BTG1 and Bcl-2.

TUNEL end staining assay. Terminal deoxynucleotidyltransferase-mediated dUTP-biotin nick-end labeling (TUNEL) assay was performed using recombinant terminal transferase (TdT) and biotin-16-dUTP (Sigma). Three groups of MDA-MB-231 xenograft sections were processed following the manufacturer's protocol, and then the stained sections were reviewed and scored using a Nikon microscope.

Statistical analysis. The results shown are the means \pm SD. A P-value $<0.05$ was considered to indicate a statistically significant difference. Statistical analyses were calculated using SPSS 17.0. Each experiment was repeated 3 times.

\section{Results}

BTG1 expression in breast cancer cell lines and clinical specimens. RT-PCR was used to assess the expression of BTG1 in human breast cancer tissues from primary tumors, lymph node metastasis, benign breast tumors and normal human breast (Fig. 1A). As assessed by RT-PCR, the BTG1 mRNA levels in benign breast tumors and normal human breast were significantly higher than levels in the primary breast tumors and lymph node metastasis tumors. We also tested a panel of breast cell lines, including normal mammary 
A
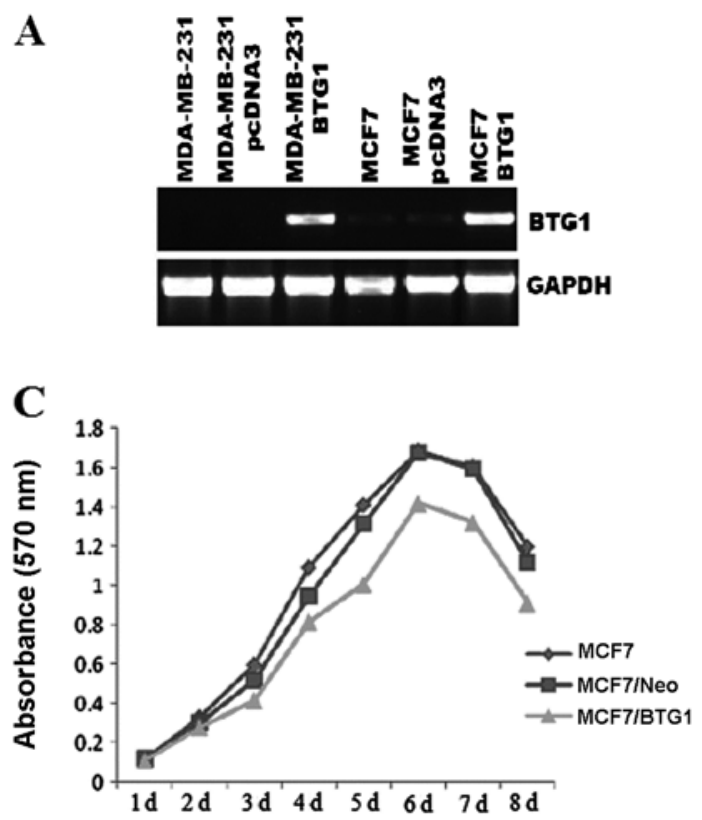

B
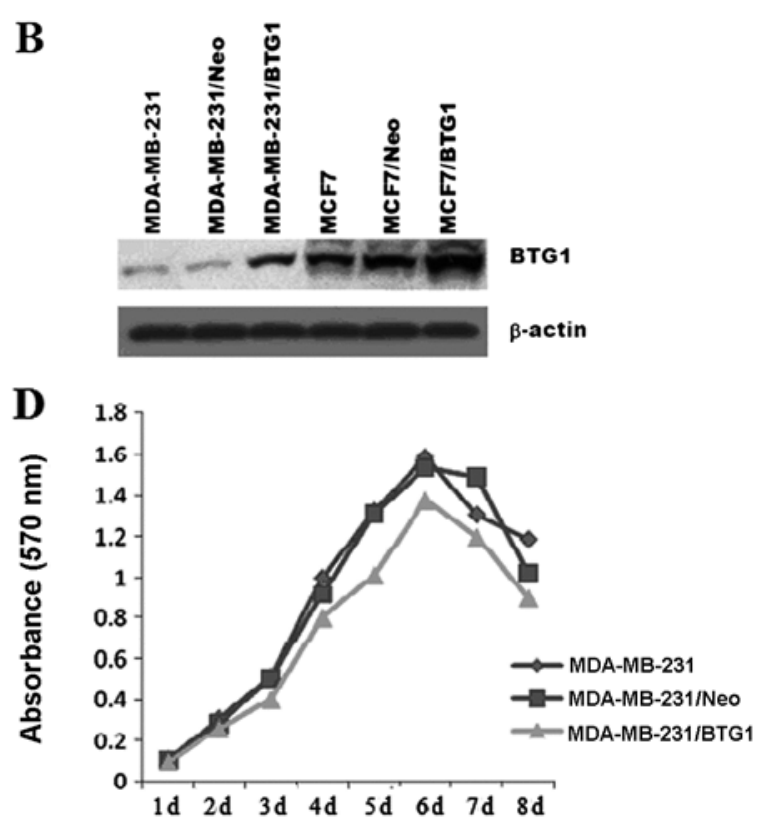

Figure 2. Overexpression of BTG1 inhibits breast cancer cell proliferation. (A) RT-PCR analysis indicating the BTG1 mRNA expression level in MCF-7 and MDA-MB-231 cells transfected with the vector control pcDNA3-neo or pcDNA3-BTG1 and their corresponding untreated cells. GAPDH levels served as the loading control. (B) Protein expression of BTG1 was analyzed by western blot analysis. $\beta$-actin levels served as the loading control. (C and D) MCF-7 and MDA-MB-231 cells and their transfectants were cultured in 96-well plates at 5x10³/well for 1, 2, 3, 4, 5, 6, 7 and 8 days. The cell growth was assessed by MTT assay as described in Materials and methods.

epithelial and vascular endothelial cell lines MCF-10A, EPH4, AG11132A, AG11134A, HBEC, HMEC, and breast cancer cell lines MCF-7, MDA-MB-23, MDA-MB-435, T47D, as shown in Fig. 1B. The western blot results revealed that the BTG1 protein was present in all the tested breast cell lines, and the BTG1 protein levels in normal mammary epithelial cells and vascular endothelial cell lines were significantly higher than levels in the breast cancer cell lines.

Overexpression of BTG1 inhibits breast cancer cell proliferation. To further explore the role of BTG1 in breast cancer, MCF-7 and MDA-MB-231 cells were transfected with DNA constructs expressing BTG1 (pcDNA3-BTG1) or a control vector (pcDNA3-neo). The G418-resistant mix clones were selected for further experiments. As shown in Fig. 2A and B, the BTG1 mRNA and protein levels in the MCF-7 and MDA-MB231 cells were measured by RT-PCR and western blot analysis, respectively. When compared with the control cells, BTG1 expression was significantly increased in the pcDN3-BTG1 sense vector-transfected MCF-7/BTG1 and MDA-MB-231/ BTG1 cells. An MTT assay was employed to determine the effect of BTG1 on cell proliferation. The untreated MCF-7 and MDA-MB-231 cells, control cells, as well as the MCF-7/ BTG1 and MDA-MB-231/BTG1 cells were grown in culture for 8 days. The cell proliferative ability of the MCF-7/BTG1 and MDA-MB-231/BTG1 cells was decreased compared with the control and untreated cells in a time-dependent manner (Fig. 2C and D).

Overexpression of BTG1 induces G0/G1 cell cycle arrest and inhibits cyclin DI and cyclin B1 expression in MCF-7 and $M D A-M B-231$ cells. We further investigated the effects of BTG1 on the cell cycle of MCF-7 and MDA-MB-231 cells.
Representative results of cell cycle distribution in untreated cells, control cells and MCF-7/BTG1 and MDA-MB-231/BTG1 cells are shown in Fig. 3A. Flow cytometric analysis revealed a statistically significant increase in the number of MCF-7/ BTG1 cells in the G0/G1 phase (22\% increase, $\mathrm{P}<0.05$; Fig. $3 \mathrm{~A})$ accompanied by a decrease in the number of cells in the $S$ and G2/M phase. In MDA-MB-231/BTG1 cells the number of cells in the G0/G1 phase was incresased (9\% increase, $\mathrm{P}<0.05$; Fig. 3A) accompanied by a decrease in the number of $\mathrm{S}$ phase cells.

Next, we evaluated the effects of BTG1 on the expression of the cell cycle proteins cyclin B1, cyclin D1, cyclin E1 and cell division cycle gene (CDC2) protein in MCF-7 and MDA-MB-231 cells using western blot analysis. As shown in Fig. 3A, overexpression of BTG1 inhibited expression of cyclin B1, cyclin D1, cyclin E1 and phosphorylated CDC2 in the MCF-7 cells. In MDA-MB-231 cells, BTG1 inhibited the expression of cyclin B1 and cyclin D1, but not cyclin E1 and phosphorylated CDC2. In summary, these results revealed that an increase in the level of BTG1 expression inhibited cyclin B1 and cyclin D1 expression in MCF-7 and MDA-MB-231 cells, which, in turn, may affect breast cancer cell (MCF-7 and MDA-MB-231) cycle distribution, and induce G0/G1 phase arrest and significantly reduce the number of $\mathrm{S}$ phase cells.

Effect of overexpression of BTG1 on breast cancer cell apoptosis and expression of Bcl-2, Bax and caspase-3. The extent of apoptosis was investigated by determining the percentage of Annexin V-stained cells, a marker of early stage apoptosis, and the apoptosis peak of flow cytometry. As determined by Annexin V assay, in MCF-7/BTG1 and MDA-MB-231/BTG1 cells, the percentage of apoptotic cells was higher than that in the control cells (Fig. 4A), and the sub-G1 peaks of flow cytometry 
$\mathbf{A}$
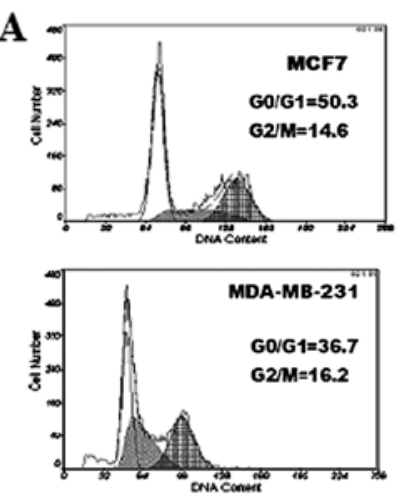
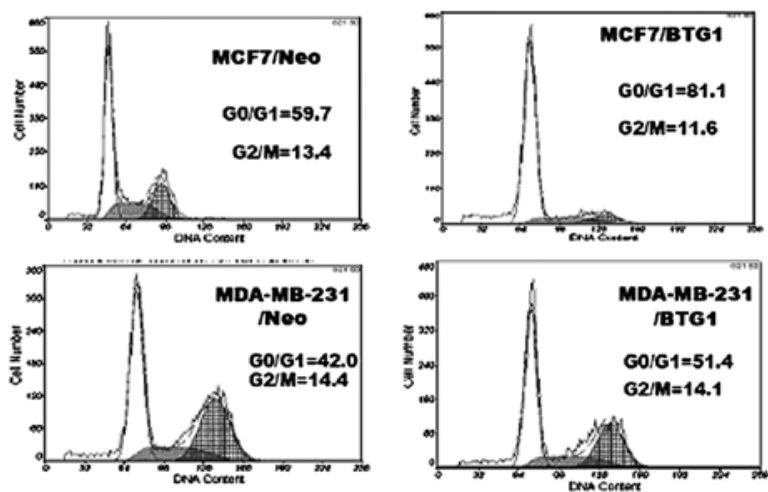

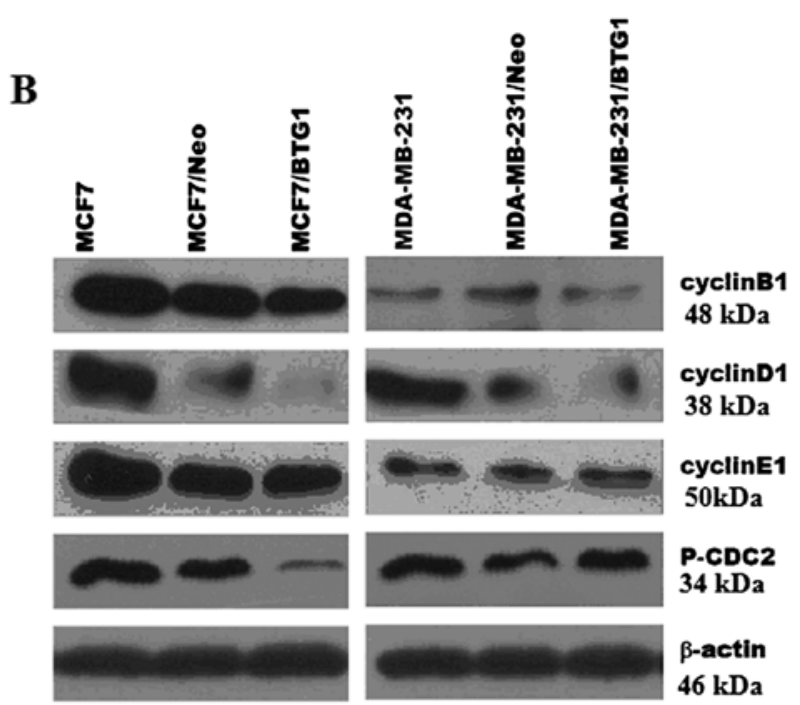

Figure 3. Overexpression of BTG1 induces G0/G1 cell cycle arrest and inhibits cyclin D1 and cyclin B1 expression. (A) After 48 h of culture, MCF-7 and MDA-MB-231 cells and their transfectants were collected to assay the cell cycle using flow cytometry as described in Materials and methods. (B) Western blot analysis indicating the expression of cyclin B1, cyclin D1, cyclin E1 and phosphorylated CDC2 protein in MCF-7 and MDA-MB-231 cells transfected with the vector control pcDNA3-neo or pcDNA3-BTG1 and their corresponding untreated cells. $\beta$-actin levels served as the loading control.

were higher than in the control cells (Fig. 4B). Next, we detected the expression of anti-apoptotic factor $\mathrm{Bcl}-2$ and pro-apoptotic factors, Bax and caspase-3, using western blot analysis. As shown in Fig. 4C, overexpression of BTG1 upregulated the expression of the anti-apoptotic protein $\mathrm{Bcl}-2$, and downregulated the expression of the pro-apoptosis proteins Bax and caspase- 3 in MCF-7 and MDA-MB-231 cells. These results indicate that the promotive effect on cell apoptosis by BTG1 is most likely mediated by Bcl-2, Bax and caspase- 3 in breast cancer cells.

Overexpression of BTG1 mediates inhibition of xenograft formation and growth in vivo. The in vitro experiments with the MCF-7 and MDA-MB-231 cells showed that the overexpression of BTG1 induced G0/G1 cell cycle arrest and cell apoptosis and inhibited cell growth. Hence, we examined whether this could also be observed in vivo.

Transfected (pcDNA3-BTG1 or negative control pcDNA3neo) and non-treated MDA-MB-231 cells were subcutaneously injected into nude mice ( $\mathrm{n}=6$ per group). After four weeks of growth, the tumor masses obtained from the MDA-MB-231/ BTG1 cell xenografts were markedly smaller than those from the control mice (Fig. 5; P<0.05). H\&E staining showed that tumor density in the MDA-MB-231/BTG1 cell tumors was deceased when compared to the control cell tumors, but no significantly higher degree of tumor necrosis and no cancer cell infiltration were noted around the tumor capsule (Fig. 6A). The immunochemical staining showed that the level of BTG1 expression was higher in the MDA-MB-231/BTG1 cell tumors (Fig. 6B), whereas the level of Bcl-2 expression was lower in the MDA-MB-231/BTG1 cell tumors (Fig. 6C). Next, we assessed tumor necrosis and apoptosis by TUNEL end staining assay. As shown in Fig. 6D, some cells exhibited nuclear pyknosis and were fragmented, irregular, inconsistent in size, and stained brownish yellow indicating cell apoptosis. Clearly, these in vivo results strongly confirmed the effect observed in vitro indicating that BTG1 plays an important role in breast cancer cell growth.

\section{Discussion}

In the present study, we examined the role of BTG1 in breast cancer cell growth. The BTG1 expression vector induced overexpression of BTG1 mRNA and protein in breast cancer cells resulted in inhibition of cell proliferation, cell G0/G1 phase arrest and cell apoptosis, which also resulted in inhibition of xenograft formation and growth in vivo. Our findings 


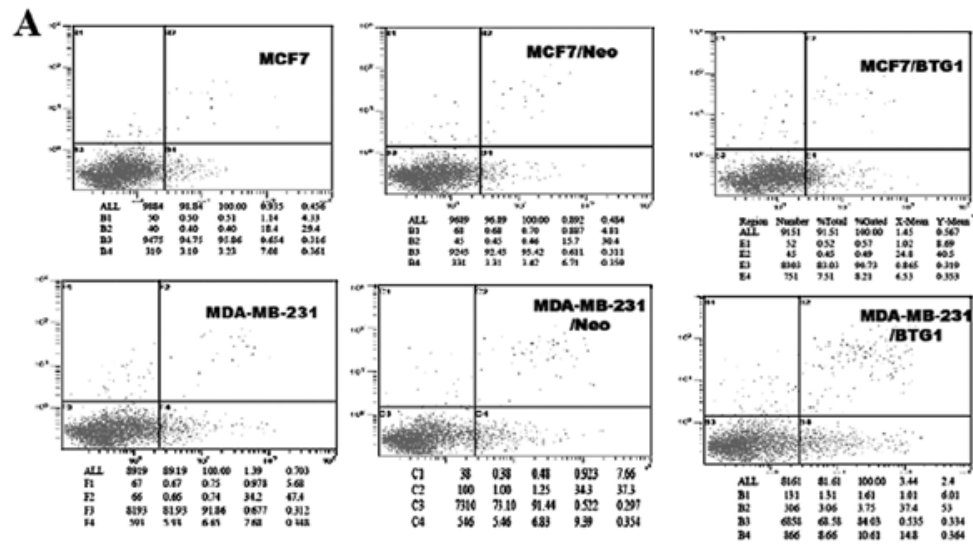

B
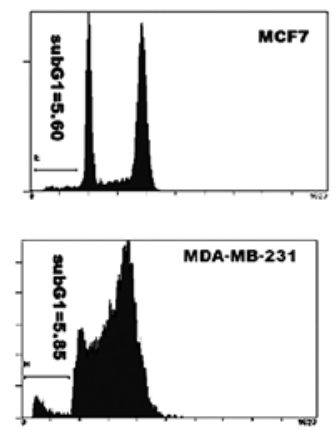
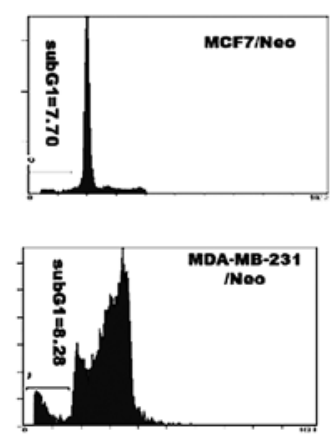

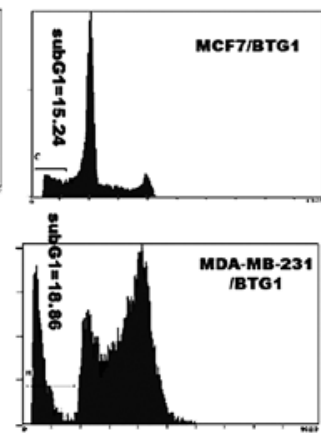

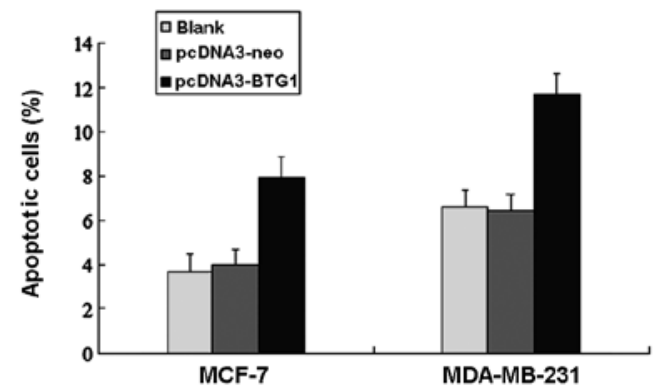

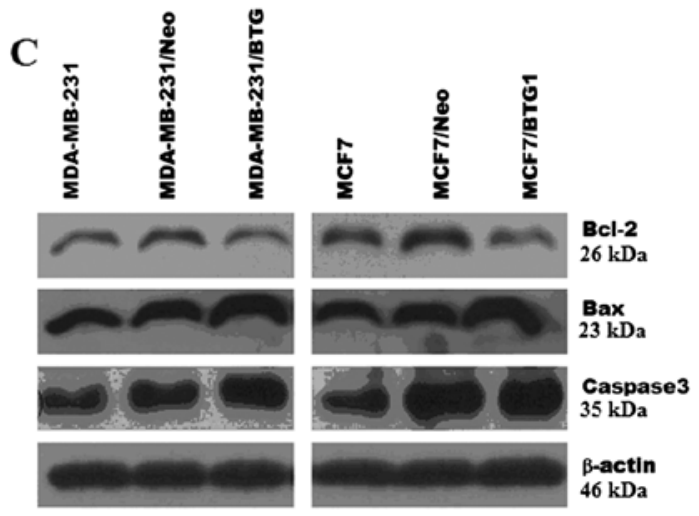

Figure 4. Effect of overexpression of BTG1 on breast cancer cell apoptosis and Bcl-2, Bax and caspase-3 expression. Annexin V-FITC assay and flow cytometry were used to detect cell apoptosis. (A) Annexin V-FITC was used to determine the percentage of apoptosis in the pcDN3-BTG1 sense vector-transfected cells (MCF-7/BTG1 and MDA-MB-231/BTG1), control cells and untreated cells. (B) Flow cytometry was used to analyze the percentage of sub-G1 peaks. (C) Western blot analysis indicating Bcl-2, Bax and caspase-3 protein level in MCF-7 and MDA-MB-231 cells transfected with the vector control pcDNA3-neo or pcDNA3-BTG1 and their corresponding untreated cells. $\beta$-actin levels served as the loading control.

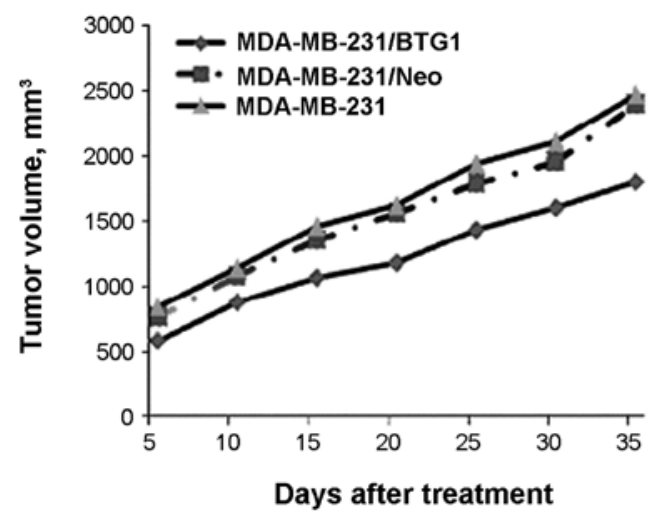

Figure 5. Effects of BTG1 overexpression on MDA-MB-231 xenografts. Growth curves of breast cancer xenografts in nude mice. Following injection of $5 \times 10^{6}$ MDA-MB-231/BTG1 cells, control cells or untreated cells, the tumor diameter was measured every five days.

demonstrated that BTG1 plays a certain role in breast cancer cell growth. Furthermore, in attempts to elucidate the mechanisms underlying the observed effects, we obtained evidence for the regulation of key genes involved in the cell cycle and cell apoptosis by BTG1.

The control of the cell cycle plays an essential role in cell growth and in the activation of important cellular processes. A large number of cells show modulated expression of molecules responsible for cell cycle arrest (15), including cyclins and cyclin-dependent protein kinases (CDKs). These molecules form the regulatory subunits (cyclins) and catalytic subunits (CDKs) of cell cycle-regulated kinases (16). Cyclin B1 and cyclin D1 both play an important role in cell cycle progression. Cyclin $\mathrm{B} 1$ expression is minimal at the initiation of $\mathrm{S}$ phase and peaks at the G2-M border; this peak in cyclin B1 activity is required for cells to enter mitosis (17); but cyclin B1 protein level is very low in the G1 phase. In addition, cyclin B1 is degraded by the ubiquitin pathway through the activation of the APC $(18,19)$; activation of the APC through specific phosphorylation of its components and the synthesis or activation of cyclin B1-directing components with the subsequent degradation of cyclin B appears to be necessary for the exit from mitosis (18). Cyclin D1 belongs to the family of D-type cyclins, which regulate G1-S cell cycle progression (20). Cyclin D1 acts through activation of CDKs that phosphorylate and inactivate the retinoblastoma protein. However, recent findings indicate that cyclin D1 also promotes cell cycle progression through CDK-independent mechanisms, such as interaction with and modulation of transcription factor activities $(21,22)$. In the present study, we found that overexpression of BTG1 affects breast cancer cell (MCF-7 and MDA-MB-231) cycle distribution, and induces G0/G1 phase arrest and significantly reduces the number of S phase cells. Furthermore, we evaluated the proteins that are directly related to cell cycle and found that overexpression of BTG1 decreased cyclin B1 and cyclin D1 protein. These results indicate that BTG1 induced G0/G1 cell cycle arrest, mediated by cyclin B1 and cyclin D1. 
A

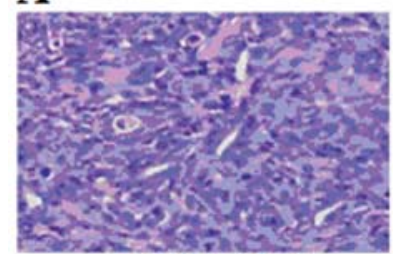

MDA-MB-231

B

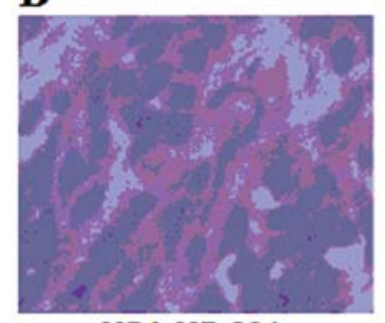

MDA-MB-231

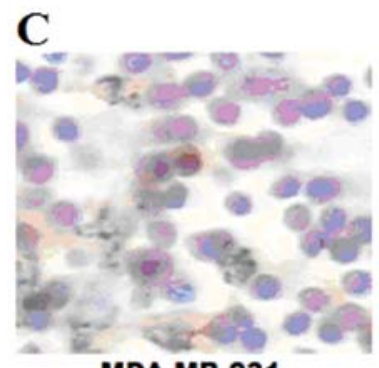

MDA-MB-231

D

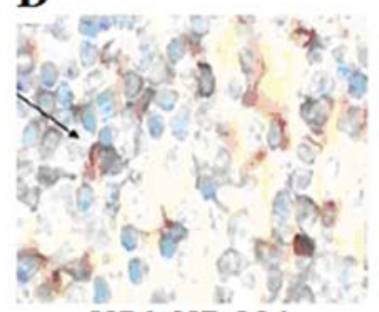

MDA-MB-231

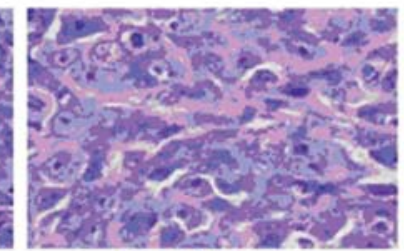

MDA-MB-231

/Neo

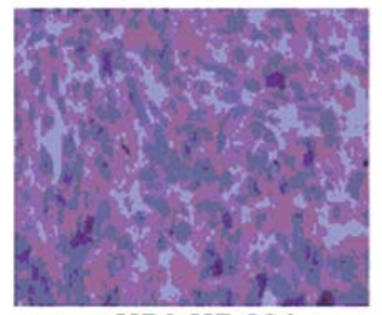

MDA-MB-231

INeo

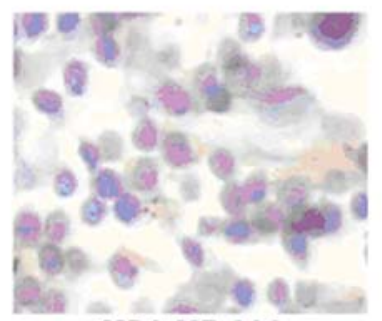

MDA-MB-231

INeo

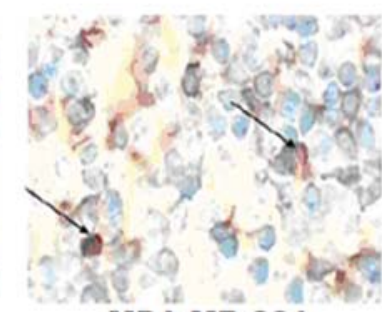

MDA-MB-231

INeo

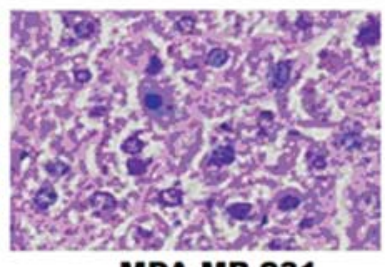

MDA-MB-231

BTG1

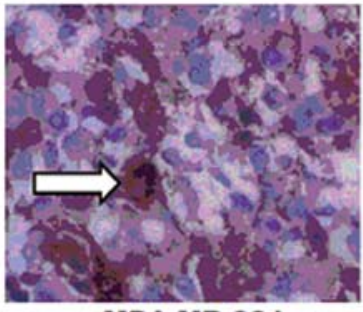

MDA-MB-231

BTG1
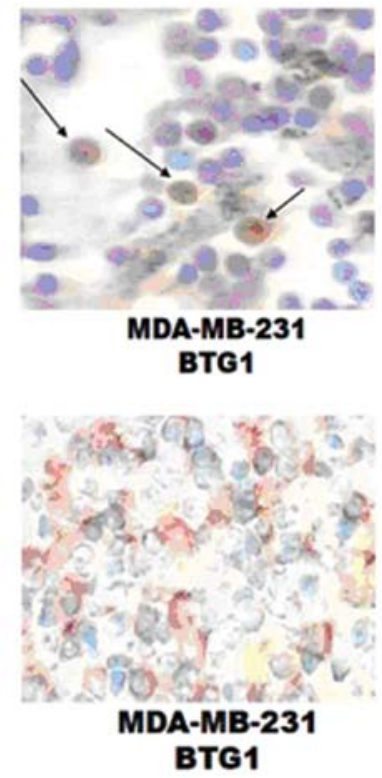

Figure 6. Histological analysis. (A) H\&E staining showed that tumor density in the MDA-MB-231/BTG1 cell tumors was deceased when compared to the control cell tumors, but no significantly higher degree of tumor necrosis and no cancer cell infiltration were noted around the tumor capsule. (B) The level of BTG1 expression was higher in the MDA-MB-231/BTG1 cell tumors and arrows indicate cells expressing BTG1. (C) There were some cells in the MDA-MB231/BTG1 cell tumors that exhibited nuclear pyknosis, and were fragmented, irregular, inconsistent in size, and arrows indicate apoptotic cells. (D) The level of Bcl-2 expression was lower in the MDA-MB-231/BTG1 cell tumors, and arrows indicate cells expressing Bcl-2.

Apoptosis occurs through two major pathways, the extrinsic or cytoplasmic pathway, which is regulated by the Fas death receptor, and the intrinsic pathway, which is controlled in part by the Bcl-2 family of proteins $(23,24)$. This family is composed of various pro-apoptotic and anti-apoptotic proteins that heterodimerize and modulate each other's function. Thus, the relative concentration of each $\mathrm{Bcl}-2$ family member is thought to determine whether cell suicide will occur. The ratio of antiapoptotic Bcl-2 to pro-apoptotic Bax is a critical determinant of apoptosis, as Bcl-2 heterodimerizes with Bax, blocking apoptosis (25). The central component of the apoptotic machinery is a proteolytic system consisting of a family of cysteinyl proteases with an absolute requirement for cleavage after aspartic acid, therefore called caspases $(26,27)$. Caspase-3 is the most intensively studied effector caspase. It has been shown that depletion of caspase-3 by homologous recombination leads to accumulation of neuronal cells whereas other tissues were not affected (28). In the present study, we demonstrated that overexpression of BTG1 induced breast cancer cell apoptosis, accompanied by a decline in Bcl-2 and an increase in Bax and caspase-3. These results indicate that the inhibitory effect on cell apoptosis by BTG1 is most likely mediated by Bcl-2, Bax and caspase- 3 in breast cancer cells.

Importantly, the finding that BTG1 inhibited breast cancer cell growth in vitro was confirmed in our animal model. The growth rate of established BTG1-upregulated xenografts was slower than the rate in the vector control group, and the immunochemical staining showed that the level of Bcl-2 expression was lower than that in the control group. Next, we found that there were many cells exhibiting nuclear pyknosis 
which were fragmented, irregular, inconsistent in size, and stained brownish yellow in the experimental group xenografts, as detected by TUNEL end staining assay. The data indicate that BTG1 induced cell apoptosis through a decrease in Bcl-2 expression, and inhibited tumor growth in vivo.

In summary, the present study investigating the role of BTG1 in breast cancer cell growth demonstrated that the protein reduced cell cycle-related proteins inducing cell cycle arrest, and altered several proteins that are directly related to cell apoptosis, and inhibited cell growth both in vitro and in vivo. These findings provide new insight into the role of BTG1 in breast cancer and may have important implication for the development of targeted therapeutics for breast cancer.

\section{Acknowledgements}

The present study was supported by the National Natural Science Foundation of China (no. 81001185), and the Universities Natural Science Foundation of Jiangsu Province (no. 10KJB310011)

\section{References}

1. Parrella P: Epigenetic signatures in breast cancer: clinical perspective. Breast Care 5: 66-73, 2010.

2. Rouault JP, Rimokh R, Tessa C, Paranhos G, Ffrench M, Duret L, Garoccio M, Germain D, Samarut J and Magaud JP: BTG1, a member of a new family of antiproliferative genes. EMBO J 11: 1663-1670, 1992.

3. Berthet C, Guehenneux F, Revol V, Samarut C, Lukaszewicz A, Dehay C, Dumontet C, Magaud JP and Rouault JP: Interaction of PRMT1 with BTG/TOB proteins in cell signalling: molecular analysis and functional aspects. Genes Cells 7: 29-39, 2002.

4. Matsuda S, Rouault J, Magaud J and Berthet C: In search of a function for the TIS21/PC3/BTG1/TOB family. FEBS Lett 497: 67-72, 2001.

5. Bakker WJ, Blazquez-Domingo M, Kolbus A, Besooyen J, Steinlein P, Beug H, Coffer PJ, Lowenberg B, von Lindern M and van Dijk TB: FoxO3a regulates erythroid differentiation and induces BTG1, an activator of protein arginine methyl transferase 1. J Cell Biol 164: 175-184, 2004

6. Hata K, Nishijima K and Mizuguchi J: Role for Btg1 and Btg2 in growth arrest of WEHI-231 cells through arginine methylation following membrane immunoglobulin engagement. Exp Cell Res 313: 2356-2366, 2007.

7. van Galen JC, Kuiper RP, van Emst L, Levers M, Tijchon E, Scheijen B, Waanders E, van Reijmersdal SV, Gilissen C, van Kessel AG, et al: BTG1 regulates glucocorticoid receptor autoinduction in acute lymphoblastic leukemia. Blood 115: 4810-4819, 2010.

8. Prevot D, Voeltzel T, Birot AM, Morel AP, Rostan MC, Magaud JP and Corbo L: The leukemia-associated protein Btg1 and the p53-regulated protein Btg2 interact with the homeoprotein Hoxb9 and enhance its transcriptional activation. J Biol Chem 275: 147-153, 2000.

9. Prevot D, Morel AP, Voeltzel T, Rostan MC, Rimokh R, Magaud JP and Corbo L: Relationships of the antiproliferative proteins BTG1 and BTG2 with CAF1, the human homolog of a component of the yeast CCR4 transcriptional complex: involvement in estrogen receptor alpha signaling pathway. J Biol Chem 276: 9640-9648, 2001.
10. Busson M, Carazo A, Seyer P, Grandemange S, Casas F, Pessemesse L, Rouault JP, Wrutniak-Cabello C and Cabello G: Coactivation of nuclear receptors and myogenic factors induces the major BTG1 influence on muscle differentiation. Oncogene 24: 1698-1710, 2005.

11. Corjay MH, Kearney MA, Munzer DA, Diamond SM and Stoltenborg JK: Antiproliferative gene BTG1 is highly expressed in apoptotic cells in macrophage-rich areas of advanced lesions in Watanabe heritable hyperlipidemic rabbit and human. Lab Invest 78: 847-858, 1998.

12. Lee H, Cha S, Lee MS, Cho GJ, Choi WS and Suk K: Role of antiproliferative B cell translocation gene-1 as an apoptotic sensitizer in activation-induced cell death of brain microglia. J Immunol 171: 5802-5811, 2003.

13. Rodier A, Marchal-Victorion S, Rochard P, Casas F, Cassar-Malek I, Rouault JP, Magaud JP, Mason DY, Wrutniak C and Cabello G: BTG1: a triiodothyronine target involved in the myogenic influence of the hormone. Exp Cell Res 249: 337-348, 1999.

14. Cho JW, Kim JJ, Park SG, Lee DH, Lee SC, Kim HJ, Park BC and Cho S: Identification of B-cell translocation gene 1 as a biomarker for monitoring the remission of acute myeloid leukemia. Proteomics 4: 3456-3463, 2004.

15. Farhana L, Dawson M, Rishi AK, Zhang Y, Van Buren E, Trivedi C, Reichert U, Fang G, Kirschner MW and Fontana JA: Cyclin B and E2F-1 expression in prostate carcinoma cells treated with the novel retinoid CD437 are regulated by the ubiquitin-mediated pathway. Cancer Res 62: 3842-3849, 2002.

16. Musgrove EA, Hamilton JA, Lee CS, Sweeney KJ, Watts CK and Sutherland RL: Growth factor, steroid, and steroid antagonist regulation of cyclin gene expression associated with changes in T-47D human breast cancer cell cycle progression. Mol Cell Biol 13: 3577-3587, 1993

17. Hwang A, McKenna WG and Muschel RJ: Cell cycle-dependent usage of transcriptional start sites. A novel mechanism for regulation of cyclin B1. J Biol Chem 273: 31505-31509, 1998.

18. Hershko A: Mechanisms and regulation of the degradation of cyclin B. Philos Trans R Soc Lond B Biol Sci 354: 1571-1576, 1999.

19. Vorlaufer E and Peters JM: Regulation of the cyclin B degradation system by an inhibitor of mitotic proteolysis. Mol Biol Cell 9: 1817-1831, 1998.

20. Quelle DE, Ashmun RA, Shurtleff SA, Kato JY, Bar-Sagi D, Roussel MF and Sherr CJ: Overexpression of mouse D-type cyclins accelerates G1 phase in rodent fibroblasts. Genes Dev 7: 1559-1571, 1993.

21. Ewen ME and Lamb J: The activities of cyclin D1 that drive tumorigenesis. Trends Mol Med 10: 158-162, 2004.

22. Arnold A and Papanikolaou A: Cyclin D1 in breast cancer pathogenesis. J Clin Oncol 23: 4215-4224, 2005.

23. Nahta R and Esteva FJ: Bcl-2 antisense oligonucleotides: a potential novel strategy for the treatment of breast cancer. Semin Oncol 30: 143-149, 2003.

24. Ghobrial IM, Witzig TE and Adjei AA: Targeting apoptosis pathways in cancer therapy. CA Cancer J Clin 55: 178-194, 2005.

25. Kymionis GD, Dimitrakakis CE, Konstadoulakis MM, Arzimanoglou I, Leandros E, Chalkiadakis G, Keramopoulos A and Michalas S: Can expression of apoptosis genes, bcl-2 and bax, predict survival and responsiveness to chemotherapy in node-negative breast cancer patients? J Surg Res 99: 161-168, 2001.

26. Cohen GM: Caspases: the executioners of apoptosis. Biochem J 326: 1-16, 1997.

27. Thornberry NA and Lazebnik Y: Caspases: enemies within. Science 281: 1312-1316, 1998.

28. Kuida K, Zheng TS, Na S, Kuan C, Yang D, Karasuyama H, Rakic P and Flavell RA: Decreased apoptosis in the brain and premature lethality in CPP32-deficient mice. Nature 384: 368-372, 1996. 